\title{
Evaluating Multi-View Representations of a Web3D Streaming Server
}

\author{
Ayat Mohammed \\ Virginia Tech \\ 3030 Torgersen Hall \\ Blacksburg, Virginia 24060 \\ maaayat@vt.edu
}

\author{
Nicholas F. Polys \\ Virginia Tech \\ 3030 Torgersen Hall \\ npolys@vt.edu
}

\author{
Vuk Marojevic \\ Virginia Tech \\ 302 Whittemore \\ maroje@vt.edu
}

\author{
Richard M. Goff \\ Virginia Tech \\ 363 Goodwin Hall \\ richgoff@vt.edu
}

\author{
Carl B. Dietrich \\ Virginia Tech \\ 302 Whittemore \\ cdietric@vt.edu
}

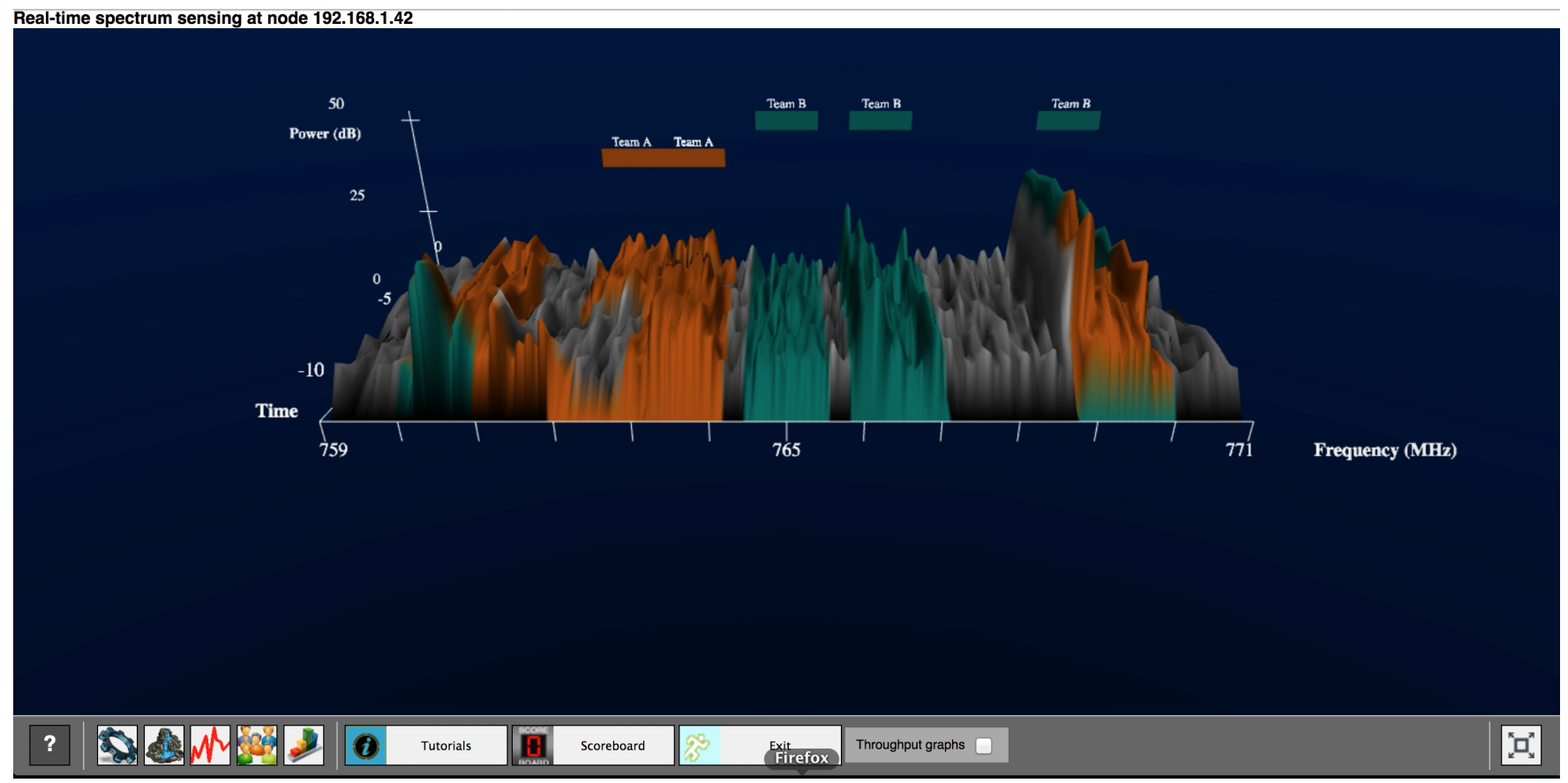

Figure 1: CORNET3D Web-based spectrum visualization.

\begin{abstract}
Analysis of multivariate data in space and time (spatio-temporal) has gained distinctive importance in all research domains. The development of new solutions that enhance massive 3D scientific visualizations on the Web is still growing. In this paper, we combine computing power, human visual perception and interaction and real-time Web3D rendering to develop a 3D visualization for

Permission to make digital or hard copies of all or part of this work for personal or classroom use is granted without fee provided that copies are not made or distributed for profit or commercial advantage and that copies bear this notice and the full citation on the first page. Copyrights for components of this work owned by others than ACM must be honored. Abstracting with credit is permitted. To copy otherwise, or republish, to post on servers or to redistribute to lists, requires prior specific permission and/or a fee. Request permissions from permissions@acm.org.

Web3D '17, Brisbane, QLD, Australia

(c) 2017 ACM. 978-1-4503-4955-0/17/06 ..\$15.00

DOI: http://dx.doi.org/10.1145/3055624.3075957
\end{abstract}

multivariate data through CORNET-3D. CORNET-3D is a software tool, which dynamically displays live wireless signal spectra, i.e. magnitudes vs. radio frequency (RF), of cognitive radio nodes in the CORNET testbed through a browser. Real time visualization of the electromagnetic spectrum activities for wireless communications provides a visually perceivable representation of the cognitive radio nodes' power and occupancy in the frequency domain. This work compares user performance between three representations (views) of multivariate scientific data to mitigate the trade-off between proximity and occlusion in CORNET-3D. The three views are Colored-Spectrum, Colored-Bars and Colored-Spectrum-with-Bars. For both types of questions (power or bandwidth), the results show that it is easy for people to carry out visual discrimination in multivariate data visualization using the Colored Spectrum-with-Bars 
view. But to get accurate judgments, the Colored spectrum view should be used.

\section{CCS CONCEPTS}

•Human-centered computing $\rightarrow$ Web-based interaction; Visual analytics; Visualization design and evaluation methods; -Computing methodologies $\rightarrow$ Appearance and texture representations;

\section{KEYWORDS}

View management, visual discrimination, 3D spectrum visualization

\section{ACM Reference format:}

Ayat Mohammed, Nicholas F. Polys, Vuk Marojevic, Richard M. Goff, and Car B. Dietrich. 2017. Evaluating Multi-View Representations of a Web3D Streaming Server. In Proceedings of Web3D '17, Brisbane, QLD, Australia, fune 05-07, 2017, 9 pages.

DOI: http://dx.doi.org/10.1145/3055624.3075957

\section{INTRODUCTION}

Analysis of multivariate data in space and time (spatio-temporal) has gained distinctive importance by researchers in all fields. The existing visualization solutions for spatio-temporal data differ based on the characteristics of the data set and have been reviewed by researchers (Andrienko et al. 2010, 2003; Zhong et al. 2012). The space dimension in spatio-temporal data can either be "stationary" or "dynamic" over time (i.e., movement data) for a certain object in the data set (Andrienko and Andrienko 2012). Adding the third axis to represent time takes us to the alternative 3D group of visualizations that combine space and time in a single display (Amini et al. 2015).

Taking into account human sensory capabilities, we can design graphic representations in such a way that important data elements and data patterns can be quickly perceived and should be represented by graphical elements that are more visually distinct than those representing less important information (Ware 2013).

Humans have predictable bias in interpreting visual-spatial information and making inferences (Shah and Carpenter 1995; Ware 2013) as well as understanding relationships over time (Michotte 1963; Ware et al. 1999). However, these biases have not been examined from a visual analytic point of view, with a focus on scientific data and user interfaces to promote understanding and insight.

According to (Elmqvist and Tsigas 2007), there are several important 'Environment Properties' relating to the configuration of objects geometry in a scene: interaction, density and complexity. Within the object interaction - spatial interaction of objects in the environment - there is proximity where objects are placed in such close proximity (without intersecting) that they occlude each other from some viewpoint. Thus when trying to visualize the many dimensions of a scientific data set, we have the same trade-off between proximity and occlusion as in IRVEs(Polys 2006; Polys et al. 2011).The pros (+) and cons (-) of common views are discussed in continuation.

(1) Small Multiples View:
(+) Provide separate screen space to represent high dimensional spaces and complex relationships among multiple variables (Elmqvist's 'proximity', Polys's 'Display Space').

(-) With small multiples we need to hold the info in the mind's working memory, which is expensive (Working memory is limited in context-switching) and errorprone.

(-) Relationships between views must be explicit.

(2) Overlay View:

(+) Collapses dimensions onto a single View (Elmqvist's 'enclosement', Polys's 'Viewport Space').

(-) Perception is difficult due to crowding/density and occlusion; also error-prone.

(3) Embedded View:

(+) Views (of dimensions) are embedded in same space as the referent (Elmqvist's 'containment', Polys's 'Object/World Space').

(-) Perception is difficult due to crowding/ density; also error-prone.

(-) Relationships between views must be explicit.

In this research, we developed and evaluated new techniques to mitigate these trade-offs and to enable analysts to better explore multivariate data sets. In this study we have quantitative and qualitative variables distributed across a spatial basis (frequency domain representation of RF signals); a similar situation to scientific visualizations where we are trying to compose several dimensions/variables into one 'view'. We call this approach:

(4) Composed View:

(+) The contribution of different attributes (layers) can be weighted and composed into the appearance of a shape (e.g. texture, color), but

(-) it may be difficult to perceive the values of or patterns in individual dimensions after they are composed.

This paper seeks to compare among three visual representations of multivariate scientific data to mitigate the trade-off between proximity and occlusion in CORNET3D. CORNET3D provides a 3D visualization of the Virginia Tech's software-defined radio (SDR) based cognitive radio network (CORNET) testbed with information on which nodes and radios are operational. The application can also display 2D and 3D plots of the spectrum, which is sensed by the radios in real time. The data is sent to the client over a WebSocket connection to enable low latency, compared to conventional HTTP (Sharakhov et al. 2014).

CORNET3D can teach students about strategies for optimal use of radio resources through a game by providing them with real-time scoring based on their choices for radio transmission parameters. CORNET3D has demonstrated that not only can Web applications provide rich portrayals of real-time sensor data, but can also serve as a 3D "serious game" platform (Sharakhov et al. 2014). The CORNET 
testbed can be used for wireless communications research, such as developing strategies to optimally utilize the limited spectral resources and enable RF coexistence among heterogeneous radio access technologies (Sohul et al. 2016). In this research, we want to improve the methods for users to visually discriminate between nodes' activities in a 3D scene in real time. The next section shows the related work to this paper.

\section{RELATED WORK}

\subsection{Space-Time Cube Based Visualization}

(Hägerstraand 1970) proposed the Space-Time Cube (STC) approach where space has two dimensions and a third dimension for the time to represent trajectory attribute data, i.e., movement data that includes other attributes. Space and time are considered inseparable and movement is described as trajectories in 3D with time being one of the coordinates. A potential problem with the STC approach is occlusion in the case where many trajectories are involved. To facilitate manipulation and perception of information, STC has been extended with interactive techniques (Gatalsky et al. 2004).

(Tominski et al. 2012) presented a new technique based on the STC with the focus on trajectory attribute data. By stacking 3D color-coded bands on a 2D map and ordering the bands based on the temporal information, the trajectories and their attributes are visualized while temporal information is directly perceivable. Extra visual cues are also added to the bands to depict direction and other properties.

An advanced version of STC enhanced with timeline as the main interaction device, time zooming or focusing, and linking of maps with corresponding symbols is presented in (Kraak 2005). The enhanced version of STC that supports many of these features has been turned into a commercial software application called GeoTime (Eccles et al. 2008; Kapler and Wright 2005).

Another drawback of the STC approach, besides occlusion, is distortion of both space and time due to a projection that makes it hard to perceive depth. Even though 3D representation of movement data has been introduced, a considerable amount of research is being devoted to finding suitable forms of representing this complex data set.

Multivariate spatio-temporal data consist of the variables time, location, and attributes that are represented in tables as data fields. Multivariate-space-time cube (MSTC) approaches 3-dimensional Cartesian coordinates to adequately represent the data of a table. The main idea is that each record of the data table is converted to a data plane of two dimensions, i.e. each data tuple of a record is shown as the graphics in a 2-dimensional domain. Then these data planes are arranged along and perpendicularly to the time axis of a 3-dimensional Cartesian coordinate system. This method includes the data of attributes in a STC to represent adequately the data fields of a table. MSTC represents visually and adequately the data of a table and offers users straight views on non-inclined maps of geographic area. MSTC is illustrated with the data table of Napoleon's 1812 Russian campaign. All data of the table is represented on a cube showing visually the great loss of soldiers as well as tragic defeat of the campaign (Tran and Nguyen 2012).

\subsection{D Visual Discrimination and Change Detection over Time}

Change blindness is defined as the difficulty to notice large changes that occur in clear view of an observer if made during an eye movement, blink, or other such disturbance (Daniel 2005; Rensink 2005). There is a distinction between the perception of a system's evolving dynamics and the perception of the system in its final state (i.e., noticing that something has changed at some time in the past, without a phenomenological experience of anything dynamic). During the perception of dynamic change, the spatiotemporal continuity of the internal representation is maintained. But, the perception of the completed change or state does not require such continuity. Basically, it could be done by a comparison of the currently visible structure with memory, using brain's working memory (Rensink 2005). To avoid this human phenomenon, researchers are developing automated and machine-learning techniques that can detect the changes when precision is needed.

\subsection{Time Series Visualization}

The typical representation of time series is line graphs. When the comparison is needed between two or more time series; a plot containing the time series is used to visually compare them. (Javed et al. 2010) evaluated graphical perception for different representations involving multiple time series through controlled laboratory experiments. Their main motivation was to provide guidelines for designers who need to find a suitable method when building a temporal visualization application. Beyond studying simple line graphs, they also include small multiples and horizon graphs. In addition, to aid perception of multiple color-coded time series, they included a novel visualization technique that they called a braided graph where filled areas are sorted in depth order for each position along the time axis.

TimeSearcher is an information visualization tool that combines timebox queries with overview displays, query-by-example facilities, and support for queries over multiple time-varying attributes (Hochheiser and Shneiderman 2004). TimeSearcher uses a tabbed display to support data sets with multiple variables. It loads the first variable in the data set as the default in a single pane of a tabbed paned window. To examine and query the values of any other variable, users select the desired variable name from the pulldown menu marked "Query Variable" in the toolbar. It also includes query manipulation tools including pattern inversion and 'leaders \& laggards' graphical bookmarks, which provide additional support for interactive exploration of data sets.

\section{CORNET-3D ENVIRONMENT}

CORNET-3D is a software tool, which dynamically visualizes live wireless spectrum using cognitive radio nodes on the web,(Fig. 1). CORNET has a total of 48 cognitive radio nodes that are installed in Kelly Hall at Virginia Tech, Blacksburg. The wireless spectra changes every unit time and it is impossible and impractical to read and understand such huge data in the span of milliseconds. Real time spectrum visualization provides a visually perceivable representation of the radios' power and bandwidth or spectrum occupancy. As the power and occupancy change with time, a Web3D 


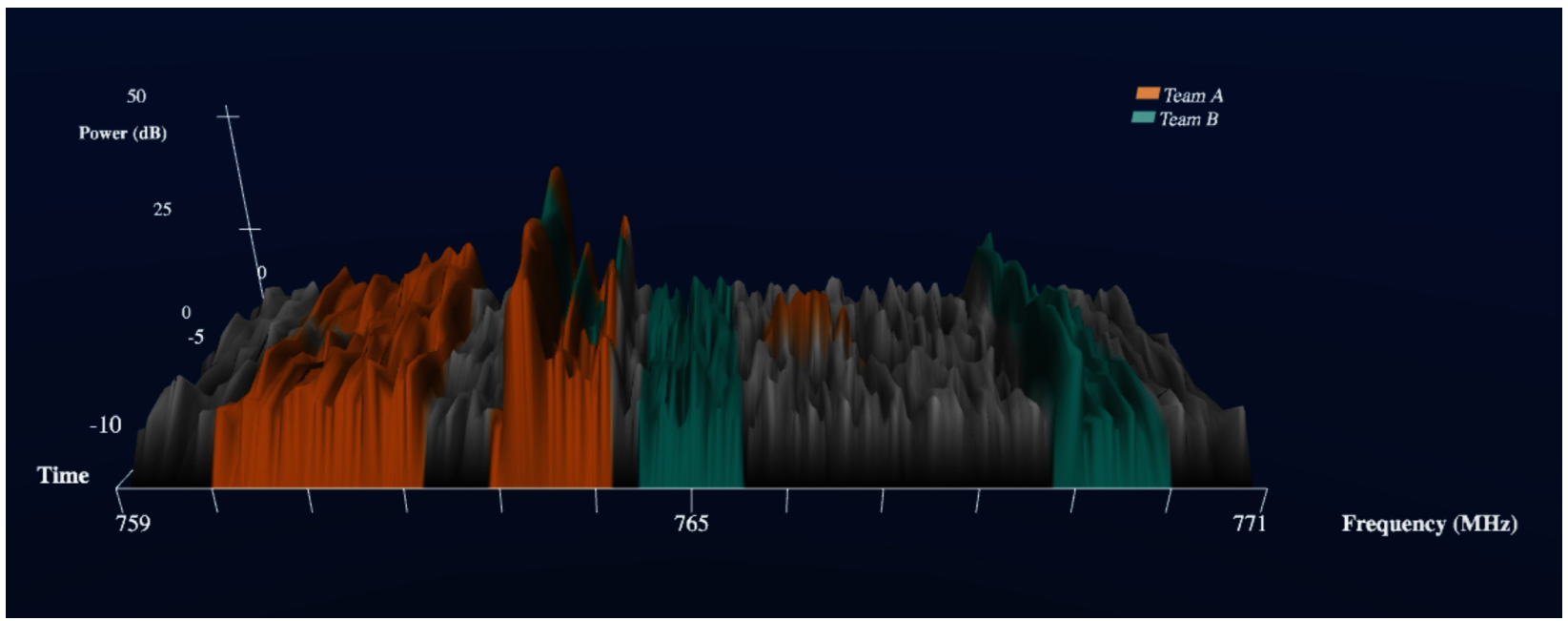

Figure 2: Colored-Spectrum view

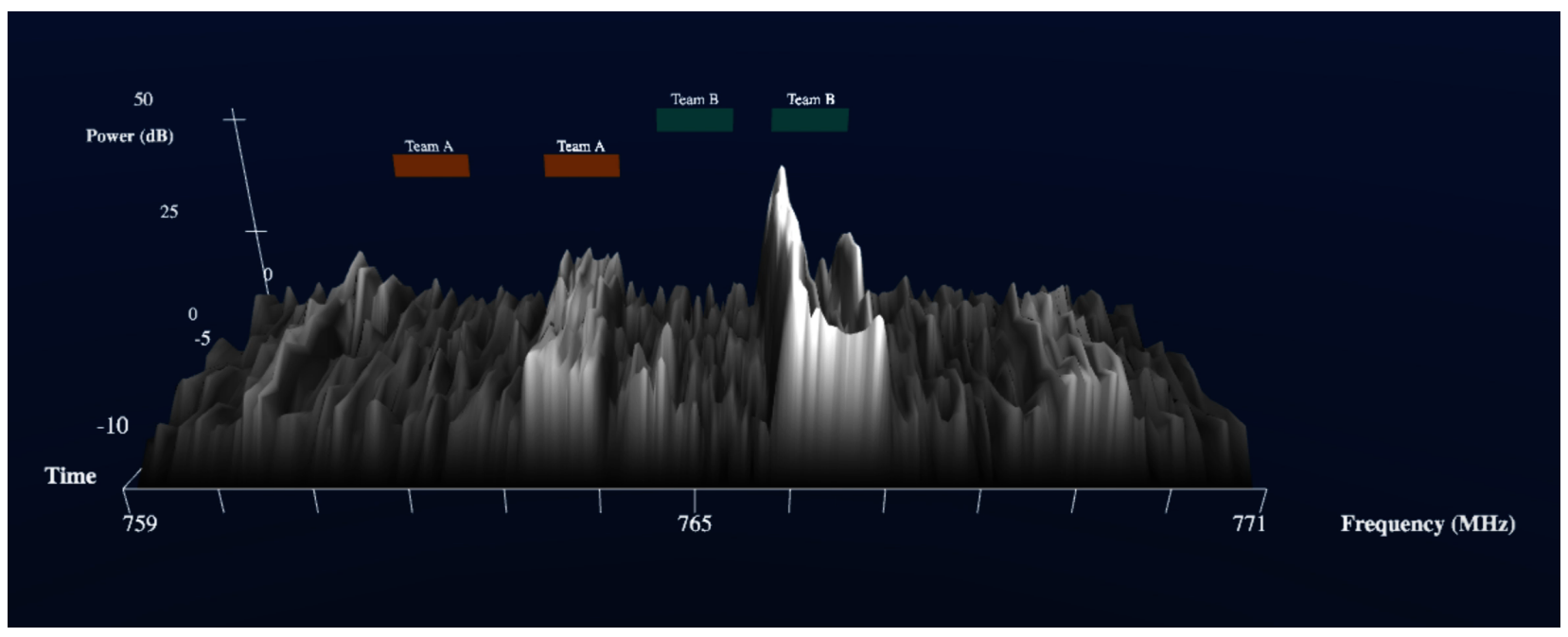

Figure 3: Colored-Bars view

visualization showing the activity of the radio node over time is utilized.

\subsection{CORNET-3D User Interface Implementation}

The user interface for CORNET-3D was implemented primarily using HTML5, CSS and JavaScript. The 3D user interactions including the floors visualization and the 3D spectrum visualization are implemented using X3DOM, while the $2 \mathrm{D}$ visualization waterfall plot is implemented through a JavaScript library, D3.js. The line graph is implemented using a JavaScript library, RGraph. The interaction with the CORNET-3D Web Server happens through socket.io JavaScript library, while all the dialogs are implemented using dialogs in jQuery UI. The Multiple CORNET-3D uses iFrames in HTML to encapsulate multiple screens from the same Web page. The $3 \mathrm{D}$ spectrum visualization is represented by the elevation grid and in another implementation by the displacement shader of the $\mathrm{X} 3 \mathrm{D}$ element plane to achieve real time rendering.

3.1.1 Shading Procedure. Signal power was received as a time series of $256 \times 1$ vector distributed on the frequency domain. The time series was used to create a canvas, with a width of 256 pixels and a length equaling the time series length. The canvas was used for the elevation grid height and the displacement shader texture. A SurfaceShaderTexture was used as a parent for the Texture element that uses the canvas for the displacement shader. The main issue with the implementation of the displacement shader, that it requires a texture of $2^{n} \times 2^{n}$ dimensions, which restricted the time series dimensions.

3.1.2 Challenge Scenario. CORNET has two main challenge scenarios, tutorial and scoreboard. In both scenarios, there are at least two radio nodes. CORNET-3D displays the scenario test 


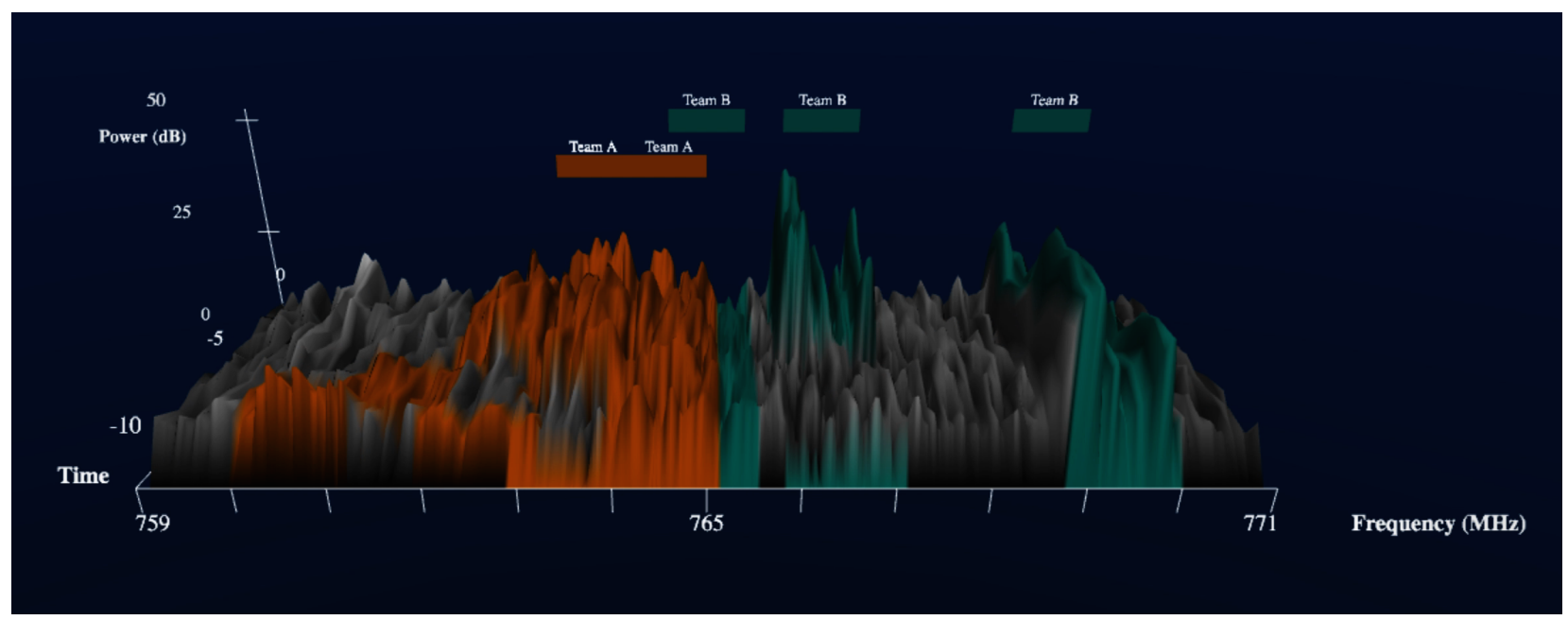

Figure 4: Colored-Spectrum-with-Bars view

parameters such as the network throughput and the operating parameters of the radio node such as its transmit power. In addition it allows users to control the radios which can be useful for tutorials (Marojevic 2015).

In this paper we use the scoreboard scenario and there were 2 teams competing to score the highest network throughput. Each team was assigned 2 nodes; also there were two interferer nodes. For experimental design purposes, in this paper we included the interferers nodes as teams' nodes. Therefore, each 3 nodes will be annotated by a different team. The 3D scene was designed to show the power, center frequency and bandwidth of each node participating in the challenge. Nodes should be labeled by the team name, which adds a new data type in our data set which is the qualitative/ categorical data type. Both quantitative and qualitative data should be mapped to appropriate visual markers in the 3D scene such as size, position, shape or color.

3.1.3 Mapping Variables to the Visual Markers in the 3D Scene. We represented the scene or spectrum activity by assigning the frequency to the $+x$-axis, the power to the $+y$-axis and the time to the $-\mathrm{z}$-axis of the $3 \mathrm{D}$ scene. The elevation grid X3D element was selected to encode the data set, with mapping the frequency to the width, the power to the height and the time to the length of the elevation grid, (Fig. 1). The bandwidth and the team annotation were represented by two techniques:

- Coloring the location of each node center frequency on the elevation grid, so the width of the colored part represents the bandwidth occupancy as in (Fig. 2), or

- Mapping to a shape(bar) size and color respectively as in (Fig.3).

For the sake of this study, we developed a third technique that combines the two techniques to test for the redundant data-visual marker mapping as in (Fig. 4).

\section{EXPERIMENTAL DESIGN}

This experiment compares among three representations of multivariate data. Therefore a 3X2 experiment for; view design (ColoredSpectrum, Colored-Bars and Colored-Spectrum-with-Bars) as in (Fig. 2, 3, 4) and signal metric (Power and Bandwidth respectively was designed according to the best practices in data visualization recommended by (Borland and Taylor II 2007; Light and Bartlein 2004; Ware et al. 1999):

- Mapping quantitative data to 'ordered' visual elements, such as position, size, and brightness.

- Mapping qualitative data, such as names and categories, to 'unordered' visual elements, such as shape and color.

The a prior hypotheses of this experiment were that:

(1) A human can visually discriminate between different values of the same quantitative measure when it is represented by an ordered visual element. Here, the signal power was represented by the luminance and the height of the elevation grid while the bandwidth was represented by the width of the bars or the spectrum colored areas.

(2) The composed view (Colored-Spectrum view) is perceptually better than the embedded view (Colored-Bars view) to visualize multivariate data.

(3) The redundant encoding of data (in the Colored-Spectrumwith-Bars view) is easier for the user but is more confusing due to visual context switching.

\subsection{Signal Detection Theory}

Signal Detection Theory provides a theoretical approach to the measurement of sensitivity and bias (Green and Swets 1988; Macmillan and Creelman 2005). It has been applied in sensory discrimination tests to provide a fundamental measure of difference, d', which is the distance between the distributions of perceptual intensity of the two sensory stimuli. This measure represents the ability of the judge to discriminate between the two products, independently 
of the test used or the cognitive strategy adopted (Macmillan and Creelman 2005).

The two-alternative forced choice (2-AFC) task is a psychophysical method, commonly used by researchers for sensory discrimination tests. 2-AFC is extracting responses from subjects about their experiences of a stimulus. Specifically, the 2-AFC experimental design is commonly used to test speed and accuracy of choices between two alternatives given a timed interval (Lu and Dosher 2013).

In order to obtain accurate evaluation of the proposed views, the user study questionnaire was designed as a 2-AFC tasks.

\subsection{User study}

This study determined the best multi-view management of multivariate data from cognitive radio nodes' spectrum as a 3-dimensional visualization. In each trial, participants were presented with a pair of images that represent a 3D visualization of CORNET3D. Subjects were given multiple-choice questions to either choose the team that scores more signal power or occupies more bandwidth in the pairs of images; also they were asked to rate the difficulty of answering those questions.

In total, the experiment had 30 samples (trials), 2 sets of 5 sessions per experimental condition. There was a set of trials asking about the signal power and another set asking about the signal bandwidth for the 3 experimental conditions:

- Colored-Spectrum view vs. Colored-Bars view (Colored vs. Bars),

- Colored-Spectrum-with-Bars view vs. Colored-Bars view (Colored-w-Bars vs. Bars), and

- Colored-Spectrum view vs. Colored-Spectrum-with-Bars view (Colored vs. Colored-w-Bars).

\subsection{Participants}

Thirty-two participants took the survey; the participants were university students ( 24 males and 8 females). Ages ranged from 18 to $34+$, and the majority was in the 18-25 range. Although only 1 of the participants answered 'yes' to the question "Have you ever experienced color blindness?" all participants passed the online test for color blindness according to (Ishihara 1987). Three participants used mobile devices to take the questionnaire. Nine wear glasses, six wear contact lenses, but the other seventeen don't use any eye wear.

\subsection{Procedures}

This study was administered as a Web survey using Virginia Tech Qualtrics. The navigation was through a Web browser as a desktop application. Each question was presented sequentially in a random order. Subjects were given multiple-choice questions to either choose the team that scores more signal power or occupies more bandwidth in the pairs of images,(Fig. 5).

They were given trial examples to help them understand the nature of the task. For each trial, they were asked to rate the difficulty of their judgment on a (5 point) scale that ranged between 'very easy' and 'very difficult'. Finally, subjects answered a question choosing one of the three views they would recommend to others. The session lasted approximately 30 minutes for each participant.

\section{RESULTS AND DISCUSSION}

\subsection{2-AFC Analysis}

Xlstat from Addinsoft was used to run 30 2-AFC tests for the 30 trials. For each set of conditions (e.g. Colored vs. Bars, Bars vs. Colored-w-Bars and Colored vs. Colored-w-Bars) there were 10 trials where 5 were asking about signal power ( 15 trials for all sets of conditions) and the other 5 were asking about the signal bandwidth (15 trials for all sets of conditions).In total, there were 12 trials (40\% of all trials) where subjects' responses showed discrimination when using the two views in comparison. But for the other $60 \%$ of the trials, participants showed the same performance in the different experimental conditions. Detailed explanation of the results of the two question types follows in continuation.

5.1.1 Trials asking about the signal power. Out of 5 trials, there were 3 trials per each set of conditions that resulted a $p-v a l u e<$ 0.05 and therefore the null hypothesis stating that d' equals to 0 was rejected. In detail, in 9 out of 15 trials ( $60 \%$ of the trials) the users' performances using the different views are coming from different normal distributions. Thus, the users' accuracy answering the questions regarding signal power using the Colored, Bars, and Colored-w-Bars is different, see (Table 1).

Table 1: 2-AFC results for questions asking about the signal power

\begin{tabular}{cccccc}
\hline \multicolumn{2}{c}{ Colored x Bars } & \multicolumn{2}{c}{ Bars x Colored-w-Bars } & \multicolumn{2}{c}{ Colored x Colored-w-Bars } \\
\hline p-value & d' & p-value & d' & p-value & d' \\
\hline 0.005 & 1.065 & $<0.0001$ & 1.428 & $<0.0001$ & 1.864 \\
$<0.0001$ & 1.839 & 0.001 & 1.098 & $<0.0001$ & 1.627 \\
0.570 & 0.000 & 0.570 & 0.000 & 0.108 & 0.451 \\
0.360 & 0.288 & 0.570 & 0.000 & 0.108 & 0.451 \\
$<0.0001$ & 1.428 & 0.001 & 1.098 & $<0.0001$ & 1.428 \\
\hline
\end{tabular}

5.1.2 Trials asking about the signal bandwidth. For Colored vs. Bars, there was only 1 trial and for Colored vs. Colored-w-Bars there were 2 trials where p-values were less than 0.05 and d' equals zero. Therefor, subjects' performances are different using these view pairs in only $3(20 \%)$ out of the 15 trials asking questions regarding signal bandwidth, see (Table 2).

Table 2: 2-AFC results for questions asking about the signal bandwidth

\begin{tabular}{cccccc}
\hline \multicolumn{2}{c}{ Colored x Bars } & \multicolumn{2}{c}{ Bars x Colored-w-Bars } & \multicolumn{2}{c}{ Colored x Colored-w-Bars } \\
\hline p-value & d' & p-value & d' & p-value & d' \\
\hline 0.640 & 0.000 & 0.430 & 0.111 & 0.004 & 0.954 \\
0.001 & 1.098 & 0.108 & 0.451 & 0.055 & 0.691 \\
0.640 & 0.000 & 0.570 & 0.000 & 0.430 & 0.111 \\
0.050 & 0.451 & 0.108 & 0.451 & 0.055 & 0.569 \\
0.570 & 0.000 & 0.570 & 0.000 & $<0.0001$ & 1.627 \\
\hline
\end{tabular}




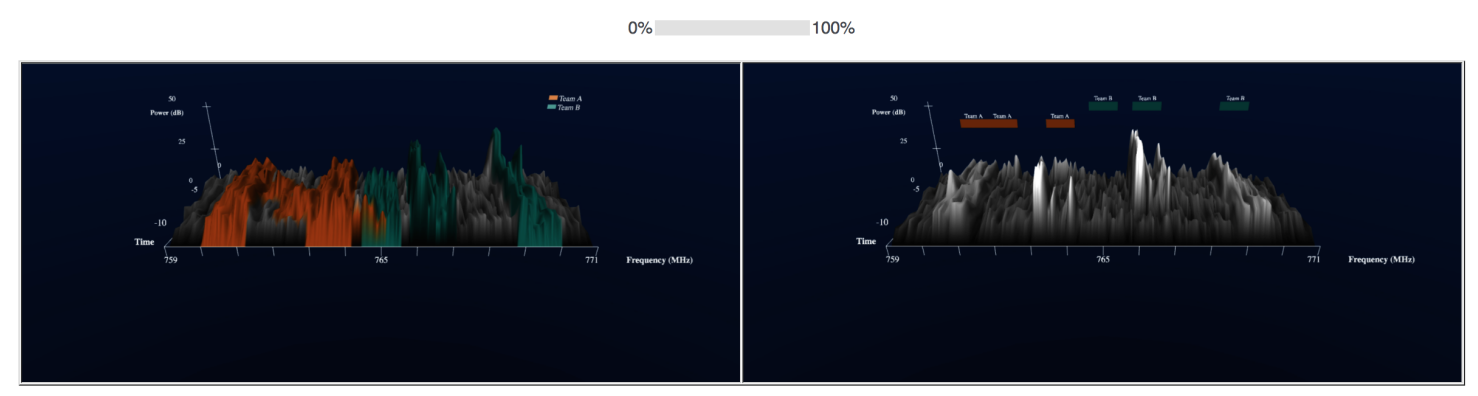

The power of a signal is represented by the height of the 3D elevation grid on the up Y-axis.

In the image on the left, which team does have more amount of power?

A

B $\quad$ Equal

Rate the difficulty of answering the previous question.

Difficult Somewhat Difficult

Neutral

Somewhat Easy

Easy

In the image on the right, which team does have more amount of power?

B

Equal

Rate the difficulty of answering the previous question.

Difficult Somewhat Difficult

Neutral

Somewhat Easy

Easy

Figure 5: Sample trial that ask users to evaluate the signal power of the two teams and rate the difficulty to answer these questions.

\subsection{ANOVA Analysis}

A 2-way ANOVA was applied afterwards to evaluate the interaction between the two independent variables, question type (signal power and signal bandwidth) and view type (Colored, Bars, and Coloredw-Bars) on subjects' performances for choosing the correct answers in all trials. With a p-value of 0.001 we concluded that the question type has a significant impact on the correctness, as the participants answers for the questions about the bandwidth were less accurate (less correctness average )than those for the power.

Also a $p$-value $<0.0001$ showed that view type has a statistical significant effect on the correctness. The interaction of the two independent variables (view type and question type) showed a significant effect on the correctness with a $\mathrm{p}$-value $=0.012$. Following are the ANOVA results of each view type as in (Fig. 6).

5.2.1 Colored view performance. With a p-value of 0.6 we concluded that there was no effect of the question type on subjects' performance in answering questions about this view and the mean values of the correct answers are nearly equal for signal power and signal bandwidth (0.838 and 0.822).

5.2.2 Bars view performance. With a $p-$ value $<0.0001$, we concluded that there was an effect of the question type on subjects' performances in answering questions about this view and the mean value of correct answers for questions asking about signal power (0.806) is statistically significantly different from the mean value of correct answers for questions asking about signal bandwidth (0.669).

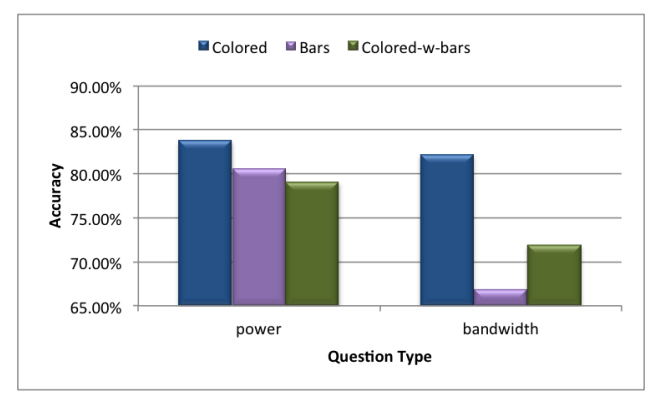

Figure 6: Results of ANOVA test to compare between the three views using the two question types.

5.2.3 Colored-w-Bars view performance. With a p-value of 0.035 , we concluded that there was an effect of the question type on subjects' performances in answering questions about this view and the mean value of correct answers for questions asking about signal power (0.791) is statistically significantly different from the mean of correct answers for questions asking about signal bandwidth (0.719).

\subsection{ROC Curves Analysis}

The diagnostic performance of a test, or the accuracy of a test to discriminate correct answers from the incorrect answers is evaluated using Receiver Operating Characteristic (ROC) curve analysis (Metz 1978; Zweig and Campbell 1993). ROC curves can also be used to 


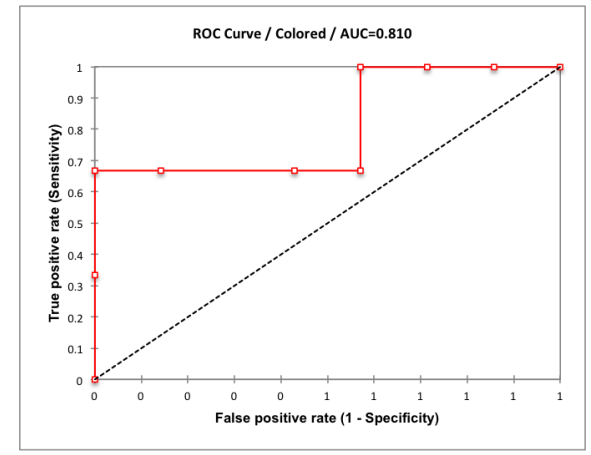

Figure 7: ROC curve for colored spectrum view for questions asking about power, $\mathrm{AUC}=\mathbf{0 . 8}$.

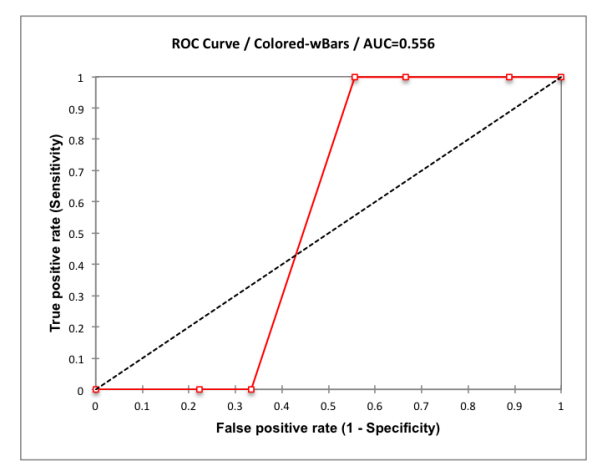

Figure 8: ROC curve for colored-with-bars view for questions asking about power, $\mathrm{AUC}=\mathbf{0 . 5 6}$.

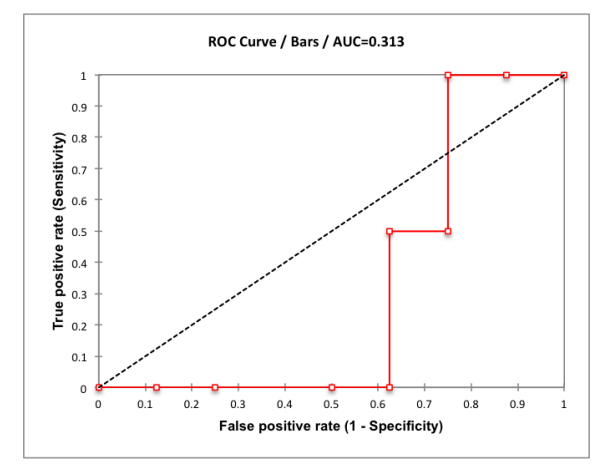

Figure 9: ROC curve for colored bars view for questions asking about power, $\mathrm{AUC}=\mathbf{0 . 3}$.

compare the diagnostic performance of two or more laboratory or diagnostic tests (Griner et al. 1981).

In a ROC curve the true positive rate (Sensitivity) is plotted as a function of the false positive rate (100-Specificity) for different cutoff points of a parameter. Each point on the ROC curve represents a sensitivity/specificity pair corresponding to a particular decision threshold. The area under the ROC curve (AUC) is a measure of how well a parameter can distinguish between two diagnostic groups (correct/incorrect).
The test that compares the AUC to 0.5 allows checking if the diagnostic test is more powerful than just a random rule. In our case, we have 3 tests corresponding to the three views. The best test is the Colored-w-Bars view but the AUC is not significantly different from 0.5. ROC Curves for questions asking about power showed that Colored view is the best test and that the AUC is significantly different from 0.5 as in (Fig. 7). (Fig. 8), 9) show the ROC curves for the other views for questions asking about power.

\subsection{ANOVA Analysis for Difficulty Rating}

A 2-way ANOVA was applied to evaluate the interaction between the two independent variables, question type (signal power and signal bandwidth) and view type (Colored, Bars, and Colored-wBars) on subjects' rating of difficulty for answering the questions in each trial. With a p-value of 0.014 we concluded that the question type has a significant impact on the difficulty rating.

Also a p-value $<0.0001$ showed that view type has a statistical significant effect on the difficulty rating. Similarly, a p-value of 0.03 showed that the interaction of the two independent variables had a significant effect on the difficulty rating. The following subsections explain the results for the three views in detail.

5.4.1 Difficulty Ratings for Colored view. With a p-value of 0.168 , we concluded that there was no effect of the question type on subjects' rating of difficulty in answering questions about this view. The means of difficulty ratings are nearly equal for signal power and signal bandwidth (2.95 and 3.07).

5.4.2 Difficulty Ratings for Bars View. With a p-value $=0.001$, we concluded that there was an effect of the question type on subjects' rating of difficulty in answering questions about this view. The mean of difficulty ratings for questions asking about signal power (2.72) is statistically significantly different from the mean of difficulty ratings for questions asking about signal bandwidth (3.05).

5.4.3 Difficulty Ratings for Colored-with-Bars View. With a pvalue of 0.577 , we concluded that there was no effect of the question type on subjects' difficulty ratings for this view. The means are nearly equal for signal power and signal bandwidth (2.64 and 2.69).

\section{CONCLUSIONS}

In this study, we designed and compared among three views for multivariate data visualization of CORNET3D (Colored Spectrum, Colored Bars and Colored Spectrum with Bars) with two types of questions (signal power and signal bandwidth). We ran a sensory discrimination user study according to the 2-AFC method to evaluate and choose among the three views.

The 2-AFC analysis showed that in $60 \%$ of the questions asking about signal power there were differences between participants' performances in the pairs of views (Colored vs. Bars, Colored vs. Colored-w-Bars and Bars vs. Colored-w-Bars). Overall, for $60 \%$ of the trials, participants showed the same performance in the different experimental conditions.

The ANOVA analysis indicated the significant effect of question type on the correctness of answering the question about the Bars and Colored-w-Bars but not for the Colored view. For difficulty rating, the question type had a significant impact on the participants' 


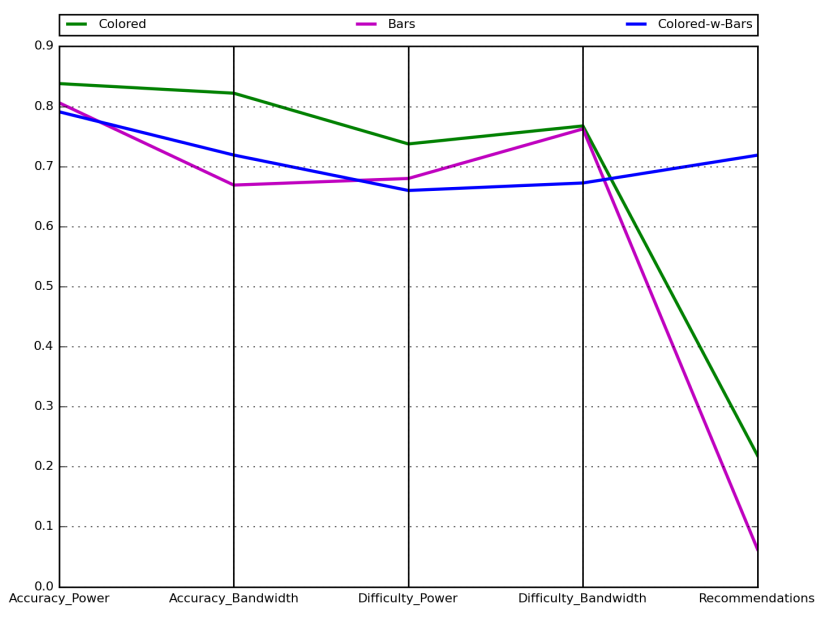

Figure 10: Recommendations, Accuracy, and Difficulty.

difficulty ratings of the Bars view only. For both the correctness and difficulty rating, there was a significant effect of the interaction between the question type and the view type.

Seventy-one percent of the participants chose the Colored-wBars view as the best view, which is consistent with the difficulty ratings of that view as the easiest for answering the questionnaire, (Fig. 10). Also this view had the largest AUC (0.664) for the ROC curve analysis.

Although the Colored view indicated the best performance for correctness and independency of question type (Power or Bandwidth), the difficulty rating of this view was neutral and it comes after the Colored-w-Bars view in the voting for the recommended view, (Fig. 10).

The Bars view showed the worst performance for correctness, high dependency on question type and had the least number of votes among the other views. Thus, this view is not recommended for this case of multivariate data visualization.

Finally, we conclude that the Colored Spectrum-with-Bars view is easier for people to carry out visual discrimination in multivariate data visualization, but for obtaining the most accurate judgments, the Colored spectrum view should be used.

\section{ACKNOWLEDGMENTS}

This work is supported in part by NSF award $\sharp 1432416$. Any opinions, findings, and conclusions or recommendations expressed in this material are those of the author(s) and do not necessarily reflect the views of the National Science Foundation. The authors would like to thank Nikhita Sharakhov and Sai Ravi Kiran for developing the initial interface.

\section{REFERENCES}

Fereshteh Amini, Sébastien Rufiange, Zahid Hossain, Quentin Ventura, Pourang Irani, and Michael J McGuffin. 2015. The impact of interactivity on comprehending 2d and $3 \mathrm{~d}$ visualizations of movement data. IEEE transactions on visualization and computer graphics 21, 1 (2015), 122-135.

Gennady Andrienko, Natalia Andrienko, Urska Demsar, Doris Dransch, Jason Dykes, Sara Irina Fabrikant, Mikael Jern, Menno-Jan Kraak, Heidrun Schumann, and Christian Tominski. 2010. Space, time and visual analytics. International fournal of
Geographical Information Science 24, 10 (2010), 1577-1600.

Natalia Andrienko and Gennady Andrienko. 2012. Visual analytics of movement: An overview of methods, tools and procedures. Information Visualization (2012), 1473871612457601.

Natalia Andrienko, Gennady Andrienko, and Peter Gatalsky. 2003. Exploratory spatiotemporal visualization: an analytical review. Journal of Visual Languages \& Computing 14, 6 (2003), 503-541.

David Borland and Russell M Taylor II. 2007. Rainbow color map (still) considered harmful. IEEE computer graphics and applications 27, 2 (2007), 14-17.

J Daniel. 2005. " Simons, and Ronald A Rensink." Change blindness: past, present, and future. (2005).

Ryan Eccles, Thomas Kapler, Robert Harper, and William Wright. 2008. Stories in geotime. Information Visualization 7, 1 (2008), 3-17.

Niklas Elmqvist and Philippas Tsigas. 2007. A taxonomy of 3D occlusion management techniques. Virtual Reality Conference 2007 (2007).

Peter Gatalsky, Natalia Andrienko, and Gennady Andrienko. 2004. Interactive analysis of event data using space-time cube. In Information Visualisation, 2004. IV 2004. Proceedings. Eighth International Conference on. IEEE, 145-152.

DM Green and JA Swets. 1988. Signal Detection Theory and Psychophysics (Peninsula, Los Altos, CA). (1988).

Paul F Griner, Raymond J Mayewski, Alvin I Mushlin, and Philip Greenland. 1981. Selection and interpretation of diagnostic tests and procedures. Principles and applications. Annals of internal medicine 94, 4 Pt 2 (1981), 557.

Torsten Hägerstraand. 1970. What about people in regional science? Papers in regional science 24, 1 (1970), 7-24.

Harry Hochheiser and Ben Shneiderman. 2004. Dynamic query tools for time series data sets: timebox widgets for interactive exploration. Information Visualization 3 , 1 (2004), 1-18.

Shinobu Ishihara. 1987. Test for colour-blindness. Kanehara Tokyo, Japan.

Waqas Javed, Bryan McDonnel, and Niklas Elmqvist. 2010. Graphical perception of multiple time series. IEEE transactions on visualization and computer graphics 16, 6 (2010), 927-934.

Thomas Kapler and William Wright. 2005. GeoTime information visualization. Information Visualization 4, 2 (2005), 136-146.

Menno-Jan Kraak. 2005. Timelines, temporal resolution, temporal zoom and time geography. In Proceedings of the 22nd International Cartographic Conference, A Coruña Spain. Citeseer.

Adam Light and Patrick J Bartlein. 2004. The end of the rainbow? Color schemes for improved data graphics. Eos 85, 40 (2004), 385-391.

Zhong-Lin Lu and Barbara Dosher. 2013. Visual psychophysics: From laboratory to theory. MIT Press.

NA Macmillan and CD Creelman. 2005. 1991Detection theory: a user's guide. Cambridge University PressMacmillanDetection theory: a user's guide1991 (2005).

Vuk Marojevic. 2015. Wireless Communication Testbed and Tools for Authentic STEM Learning. (2015).

Charles E Metz. 1978. Basic principles of ROC analysis. In Seminars in nuclear medicine, Vol. 8. Elsevier, 283-298.

Albert Michotte. 1963. The perception of causality. (1963).

Nicholas F. Polys. 2006. Display techniques in information-rich virtual environments. Diss. Virginia Polytechnic Institute and State University 2006 (2006).

Nicholas F Polys, Doug A Bowman, and Chris North. 2011. The role of depth and gestalt cues in information-rich virtual environments. International fournal of Human-Computer Studies 69, 1 (2011), 30-51.

Ronald A Rensink. 2005. Change blindness. Neurobiology of attention (2005), 76-81.

Priti Shah and Patricia A Carpenter. 1995. Conceptual limitations in comprehending line graphs. Fournal of Experimental Psychology: General 124, 1 (1995), 43.

Nikita Sharakhov, Vuk Marojevic, Ferdinando Romano, Nicholas Polys, and Carl Dietrich. 2014. Visualizing real-time radio spectrum access with CORNET3D. In Proceedings of the 19th International ACM Conference on 3D Web Technologies. ACM, 109-116.

Munawwar M Sohul, Miao Yao, Xiaofu Ma, Eyosias Y Imana, Vuk Marojevic, and Jeffrey H Reed. 2016. Next generation public safety networks: a spectrum sharing approach. IEEE Communications Magazine 54, 3 (2016), 30-36.

Christian Tominski, Heidrun Schumann, Gennady Andrienko, and Natalia Andrienko. 2012. Stacking-based visualization of trajectory attribute data. IEEE Transactions on visualization and Computer Graphics 18, 12 (2012), 2565-2574.

V. P. Tran and T. H. Nguyen. 2012. Multivariate-space-time cube to visualize multivariate data. International fournal of Geoinformatics 8, 4 (2012), 2012.

Colin Ware. 2013. Information visualization: perception for design. Elsevier.

Colin Ware, Eric Neufeld, and Lyn Bartram. 1999. Visualizing causal relations. In Proceedings of IEEE Information Visualization, Vol. 99.

Chen Zhong, Tao Wang, Wei Zeng, and Stefan Müller Arisona. 2012. Spatiotemporal visualisation: A survey and outlook. In Digital Urban Modeling and Simulation. Springer, 299-317.

Mark H Zweig and Gregory Campbell. 1993. Receiver-operating characteristic (ROC) plots: a fundamental evaluation tool in clinical medicine. Clinical chemistry 39, 4 (1993), 561-577. 